\title{
Serous microcystic adenoma of the pancreatic head: Report of two cases and review of the literature
}

\author{
JIARUI PU ${ }^{1 *}$, QIANGSONG TONG ${ }^{*}$, CHENGKAI ZHOU $^{2}$, XIUPING YANG ${ }^{3}$ and LIDUAN ZHENG $^{3}$ \\ Departments of ${ }^{1}$ Surgery, ${ }^{2}$ Radiology and ${ }^{3}$ Pathology, Union Hospital of Tongji Medical College, \\ Huazhong University of Science and Technology, Wuhan 430022, Hubei, P.R. China
}

Received February 9, 2010; Accepted April 15, 2010

DOI: 10.3892/ol_00000126

\begin{abstract}
Serous microcystic adenomas (SMAs), also known as glycogen-rich cystadenomas, are uncommon exocrine tumors mainly involved in the body and tail of the pancreas. In this study, we report two SMA cases of the pancreatic head. In case 1, a 47-year-old woman was referred to our hospital for abdominal bloating and back pain; in case 2, a pancreatic space-occupying lesion was incidentally discovered in an asymptomatic 71-year-old female during an ultrasound. In both cases, a computer tomography scan showed well-demarcated and multilocular cysts in the head of the pancreas, and a central scar and calcifications were noted in case 1. During the exploratory laparotomies, the multiple cystic masses were found in the pancreatic head. A segmental resection of the pancreas and pancreatic jejunal anastomosis were performed. Histologically, the tumors were composed of tiny cysts that were filled with clear fluid and lined by a single layer of cuboidal cells with round, centrally located nuclei and clear cytoplasm, without cellular atypia. Immunohistochemical studies showed that the neoplastic cells were positive for cytokeratin 7 and vimentin but negative for synaptophysin and cytokeratin 20. A postoperative follow-up indicated no recurrence in the patients. We reviewed the literature regarding the etiology, clinical presentation, imaging characteristics, features and management of this lesion.
\end{abstract}

\section{Introduction}

Serous microcystic adenomas (SMAs) are rare tumors in the pancreas that are traditionally regarded as benign lesions with no or little malignant potential (1). SMAs occur mainly

Correspondence to: Dr Liduan Zheng, Department of Pathology, Union Hospital of Tongji Medical College, Huazhong University of Science and Technology, Wuhan 430022, Hubei, P.R. China

E-mail: ld_zheng@hotmail.com

*Contributed equally

Key words: serous microcystic adenoma, pancreatic head, diagnosis, managements in elderly women. The majority of patients are asymptomatic and the tumors are only detected incidentally (2). The typical appearance of a SMA on computed tomography (CT) is a well-demarcated, lobulated cystic lesion with multiple thin septations. A central stellate scar is observed at the center of the lesion in certain cases. Microscopic examination reveals that the cysts are lined by a single layer of cuboidal or flattened cells, and the cytoplasm is positive for Periodic acid-Schiff (PAS) staining. The preoperative diagnosis is mainly based on a CT scan or an ultrasound. The differential diagnosis between SMAs and other non-neoplastic and neoplastic cysts is significant due to the considerable difference in their management (3). Pancreatic pseudocysts occupy $80-90 \%$ of pancreatic cystic lesions, and are associated with histories of pancreatitis (4). Mucinous cystic neoplasms (MCN), intraductal papillary-mucinous neoplasms (IPMN) and solid pseudopapillary neoplasms (SPPN) are common cystic neoplasms of the pancreas that have the potential to transform into malignant tumors $(1,5,6)$. Other rare cystic lesions of the pancreas include pancreatic endocrine neoplasms, acinar cell cystadenomas, enterogenous cysts, cystic mesenchymal tumors, endometrial cysts and parasitic cysts $(7,8)$. We present two female cases of SMA and review the literature regarding its etiology, clinical presentation, imaging characteristics, histopathological features and management.

\section{Case reports}

Case 1. A 47-year-old woman was admitted to our hospital with abdominal bloating and back pain, but without nausea, vomiting, jaundice, melena or fever. She had undergone a left radical mastectomy 10 years previously. No irregularities were noted in the physical examination. The complete blood count, biochemical parameters and tumor biomarkers were normal. A CT scan revealed a multiloculated cystic mass, $4.1 \times 3.3 \mathrm{~cm}^{2}$ in size, with a central scar and calcifications in the pancreatic head (Fig. 1A and B). An enhanced CT scan showed that the body and tail of the pancreas were normal, without expansion of the pancreatic ducts or lymphadenopathy (Fig. 1C). During an exploratory laparotomy, a multiple cystic mass was located in the pancreatic head. An intraoperative frozen section revealed a SMA. Segmental resection of the pancreas and pancreatico-jejunal anastomosis were performed. A post- 

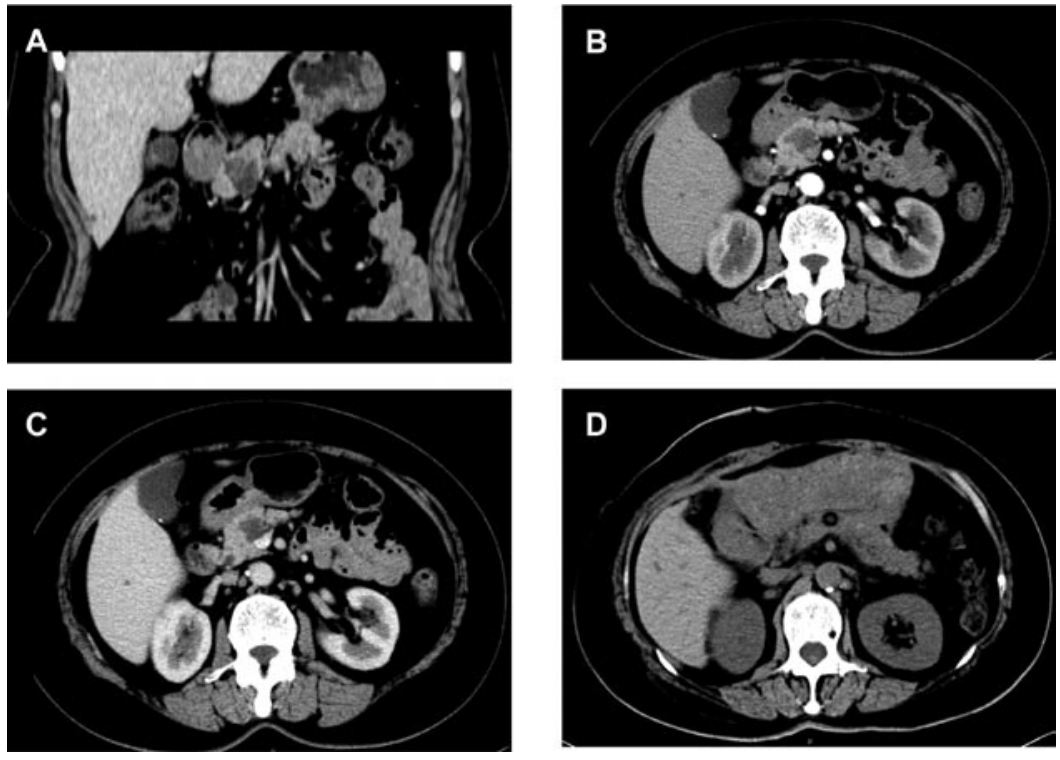

Figure 1. Computer tomography (CT) scan of the abdomen showing a mass in the head of pancreas in case 1. (A and B) A multiloculated cystic mass, $4.1 \times 3.3 \mathrm{~cm}^{2}$ in size, was noted in the pancreatic head. A central scar and calcifications were also observed. (C) An enhanced CT scan showed that the body and tail of the pancreas were normal, without expansion of the pancreatic ducts or lymphadenopathy. No increased density of tumor was observed. (D) A postoperative $\mathrm{CT}$ scan indicated the remnant pancreas and no tumor recurrence.
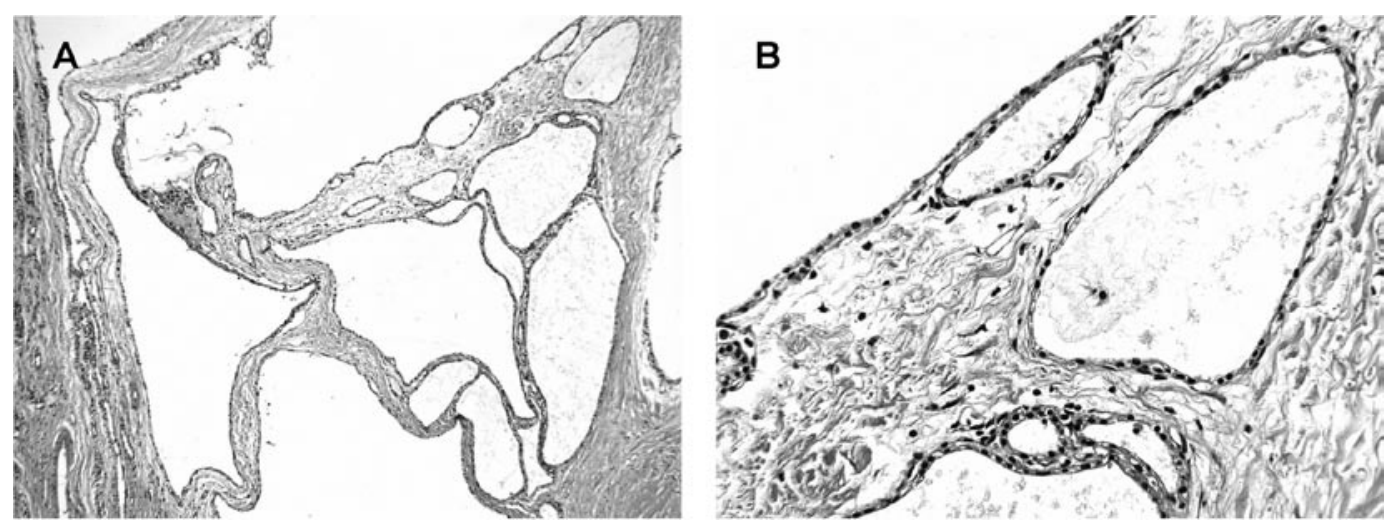

Figure 2. Histopathological features of the serous microcystic adenoma in case 1. Microscopic examination with hematoxylin and eosin staining revealed that the cysts were lined with a single layer of cuboidal cells without atypia. (A) Original magnification, x100. (B) Original magnification, x400.

operative CT scan indicated no tumor recurrence (Fig. 1D). Grossly, the resected mass had a central scar surrounded by tiny loculi filled with clear fluid. Stellate calcifications were also noted. A microscopic examination with hematoxylin and eosin $(\mathrm{H} \& \mathrm{E})$ staining revealed that the cysts were lined with a single layer of cuboidal cells without atypia (Fig. 2), and the cytoplasm was positive for PAS staining (data not shown). Two pathologists confirmed the SMA diagnosis.

Case 2. A pancreatic space-occupying lesion was incidentally discovered by ultrasound during a routine physical examination of a 71-year-old female. She was referred to our hospital for further investigation and treatment. The patient was asymptomatic on admission. The patient had exhibited a history of hypertension for more than a decade, and her blood pressure was kept under control by medication. The physical examination was negative. The complete blood count, biochemical parameters, and tumor biomarkers were normal.
A CT scan showed an irregular and multiple cystic mass, $2 \times 2 \mathrm{~cm}^{2}$ in size, in the head of pancreas (Fig. 3A, B and C). An F-18-fluorodeoxyglucose positron emission tomography (FDG-PET) showed a low-density area with a mildly increased metabolism in the pancreatic head. During the segmental resection of pancreas, a well-demarcated mass was found. An intraoperative frozen section revealed a SMA. Subsequently, pancreatico-jejunal anastomosis was performed. A postoperative CT scan indicated no recurrence of the tumor (Fig. 3D). Grossly, the resected mass had a central scar and multiple small cysts filled with clear fluid. A pathological examination with H\&E staining showed a SMA with the reactive hyperplasia of peripheral lymph nodes (Fig. 4). The cytoplasm of neoplastic cells was positive for PAS staining (data not shown). Two pathologists confirmed the SMA diagnosis.

Immunohistochemical staining. Immunohistochemistry was performed using the avidin-biotin immunoperoxidase method 

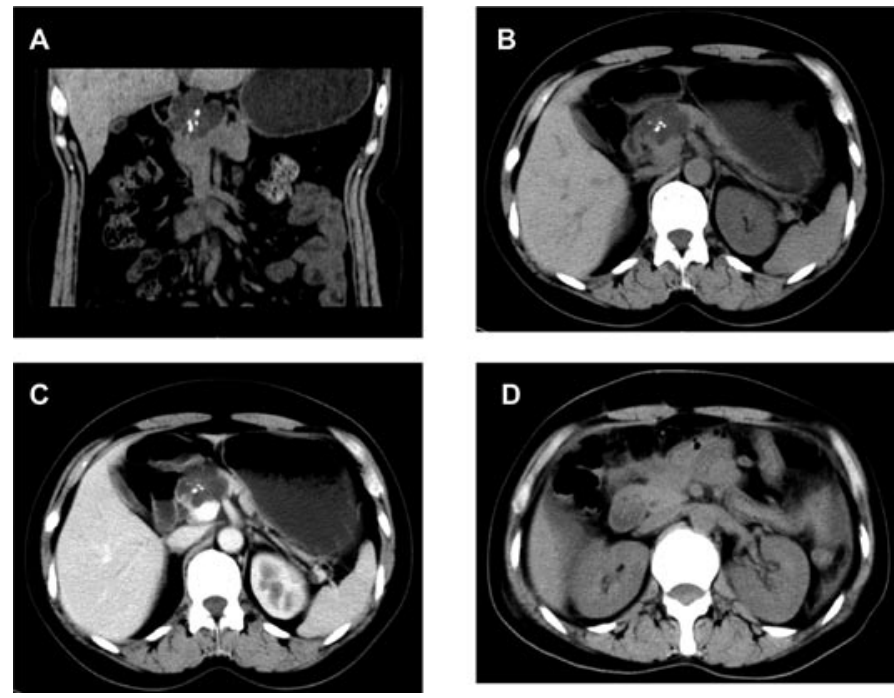

Figure 3. Computer tomography (CT) scan of the abdomen showing a mass in the pancreatic head in case 2. (A and B) An irregular solid-cystic mass, $2 \times 2 \mathrm{~cm}^{2}$ in size, was noted in the pancreatic head. A central scar and calcifications were also observed. (C) An enhanced CT scan showed no increased tumor density. (D) A postoperative CT scan indicated the normal body and tail of the pancreas but no tumor recurrence.
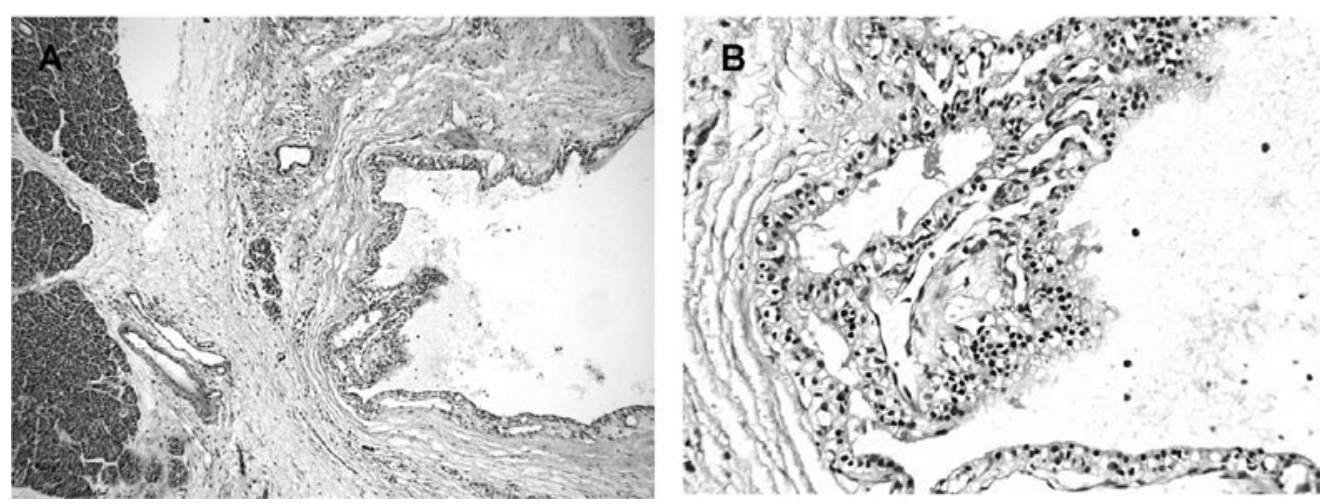

Figure 4. Histopathological features of the serous microcystic adenoma in case 2. Microscopic examination with hematoxylin and eosin staining revealed that the epithelial lining of the cysts is composed of low cuboidal cells with clear cytoplasm. No cellular atypia was observed. (A) Original magnification, x100. (B) Original magnification, $\mathrm{x} 400$.
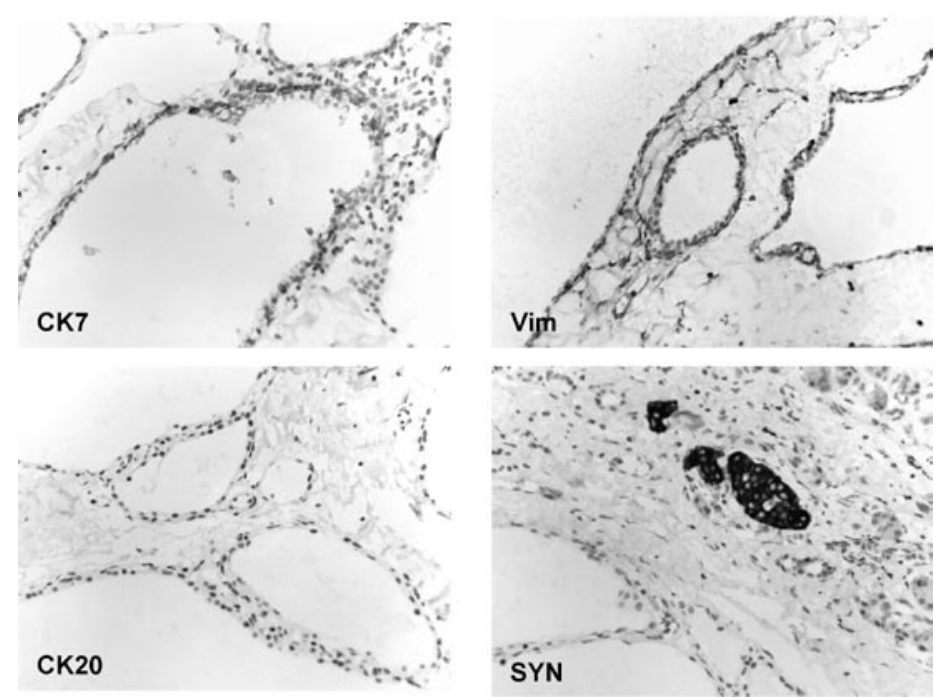

Figure 5. Immunohistochemical studies of serous microcystic adenomas. Immunohistochemistry was performed using the avidin-biotin immunoperoxidase method. The results showed that the neoplastic cells were positive for cytokeratin 7 (CK7) and vimentin (Vim), but negative for cytokeratin 20 (CK20) or synaptophysin (SYN). Original magnification, $\mathrm{x} 400$. 
Table I. Characteristics of pancreatic cystic lesions.

\begin{tabular}{|c|c|c|c|c|c|}
\hline Type & SMA & $\mathrm{MCN}$ & IPMN & SPPN & Pseudocyst \\
\hline Malignant potential & Rarely & Yes & Yes & Yes & No \\
\hline \multicolumn{6}{|l|}{ Clinical features } \\
\hline Gender & $\mathrm{F}>\mathrm{M}$ & $\mathrm{F}>\mathrm{M}$ & $\mathrm{M}>\mathrm{F}$ & $\mathrm{F}>\mathrm{M}$ & $\mathrm{M}>\mathrm{F}$ \\
\hline Age & Elderly & Middle-aged & Elderly & Young & Variable \\
\hline Pancreatitis history & No & No & Yes & No & Yes \\
\hline Symptoms & $\begin{array}{l}\text { Asymptomatic } \\
\text { mostly }\end{array}$ & $\begin{array}{l}\text { Abdominal pain, } \\
\text { weight loss }\end{array}$ & $\begin{array}{l}\text { Vague } \\
\text { abdominal } \\
\text { discomfort }\end{array}$ & $\begin{array}{c}\text { Abdominal pain, } \\
\text { weight loss, } \\
\text { palpable mass }\end{array}$ & $\begin{array}{l}\text { Abdominal pain, } \\
\text { gastric outlet } \\
\text { obstruction, } \\
\text { nausea }\end{array}$ \\
\hline Imaging findings & $\begin{array}{l}\text { Well-delineated } \\
\text { mass with } \\
\text { small cysts and } \\
\text { central scar }\end{array}$ & $\begin{array}{l}\text { Single cyst, } \\
\text { thickened wall }\end{array}$ & $\begin{array}{c}\text { Thin capsule } \\
\text { communicated } \\
\text { with the } \\
\text { pancreatic duct }\end{array}$ & $\begin{array}{c}\text { Solid mass } \\
\text { without septa }\end{array}$ & $\begin{array}{l}\text { Single cyst, } \\
\text { thin wall }\end{array}$ \\
\hline $\begin{array}{l}\text { Histopathological } \\
\text { features }\end{array}$ & $\begin{array}{l}\text { Cysts composed } \\
\text { of low-cuboidal, } \\
\text { small cells with } \\
\text { clear cytoplasm }\end{array}$ & $\begin{array}{l}\text { Flat mucinous- } \\
\text { type epithelium }\end{array}$ & $\begin{array}{c}\text { Flat/papillary } \\
\text { mucinous-type } \\
\text { epithelium }\end{array}$ & $\begin{array}{l}\text { Pseudopapillary } \\
\text { neoplasm with } \\
\text { tumor cells } \\
\text { arranged around } \\
\text { capillaries }\end{array}$ & $\begin{array}{l}\text { Necrotic fat and } \\
\text { a combination of } \\
\text { necrotic cells }\end{array}$ \\
\hline Management & $\begin{array}{l}\text { Resection/ } \\
\text { observation }\end{array}$ & Resection & $\begin{array}{l}\text { Resection/ } \\
\text { observation }\end{array}$ & Resection & $\begin{array}{c}\text { Surgical drainage/ } \\
\text { observation }\end{array}$ \\
\hline
\end{tabular}

SMA, serous microcystic adenoma; MCN, mucinous cystic neoplasms; IPMN, intraductal papillary-mucinous neoplasms; SPPN, solid pseudopapillary neoplasms.

in both cases. As shown in Fig. 5, the neoplastic cells were positive for cytokeratin 7 (CK7, OV-TL 12/30 clone, Dako, Glostrup, Denmark) and vimentin (Vim, V9 clone, Dako), but negative for cytokeratin 20 (CK20, Ks20.8 clone, Dako) and synaptophysin (SYN, SY38 clone, Dako).

\section{Discussion}

SMAs are uncommon cystic tumors of the pancreas and constitute $1-2 \%$ of all exocrine pancreatic neoplasms $(2,9)$. The age range of patients was 24-91 years (mean age 66) (6). SMAs mainly occur in elderly women, and the female-tomale ratio is 3:1 $(2,6)$. As shown in Table I, MCN and SPPN tend to occur in females. In contrast, the incidence of IPMN is higher in men (6). The etiology and pathogenesis of SMAs remains largely unknown. SMAs are traditionally considered to be benign; however, malignant transformation has been noted (10). It has been reported that $29-47 \%$ of patients are asymptomatic and that the tumors are detected incidentally $(1,11,12)$. Patients with symptoms usually manifest abdominal pain, weight loss, nausea, vomiting, fever and a palpable abdominal mass $(2,12)$. If the tumors are large, they may result in obstructive symptoms such as jaundice. Portal hypertension, hemoperitoneum, and acute gastrointestinal hemorrhage are rare clinical manifestations (6). In this study, one patient presented with abdominal bloating and back pain, while the other was asymptomatic. We believe that the diagnosis of SMAs should be kept in mind even though their clinical manifestations are non-specific.
The radiological appearance of SMA is a well-demarcated mass composed of multiple small cysts, usually located in the body and tail of pancreas. However, in the two cases studied herein, the cystic mass was located in the pancreatic head. The small cysts are less than $2 \mathrm{~cm}$ in diameter. Central calcified scars were observed in approximately $30 \%$ of cases. No communication with the pancreatic duct was noted in SMAs (9). No enhancement of the tumor was observed on enhanced CT scans of SMAs. In contrast, IPMN exhibited a thin capsule and often communicated with the pancreatic ducts, while SPPNs were considered to be solid and cystic lesions. It is difficult to preoperatively distinguish between SMAs and MCNs using radiologic methods (Table I). In magnetic resonance imaging, SMAs exhibited a low signal intensity on T1-weighted images and a high signal intensity on T2-weighted images (7). Endoscopic ultrasonography in conjunction with fine needle aspiration of cyst fluid is feasible as well. The carcinoembryonic antigen values of cyst fluid are universally low in SMAs, and are generally elevated in mucinous lesions and cystadenocarcinomas (11). We believe that the preoperative diagnosis of a SMA should be considered when ultrasounds and CT scans indicate a well-delineated mass with multiple small septations. However, it is difficult to distinguish a benign serous cystadenoma from a malignant serous cystadenocarcinoma, since imaging examinations and core needle biopsy are not entirely credible (10). On the other hand, the intraoperative frozen section is useful for the correct diagnosis of SMAs. 
Grossly, the cyst fluid of SMAs is clear, thin, watery and straw-colored. In contrast, MCNs contain thick and viscous fluid. In the center of SMAs, fibrous scars are surrounded by many tiny cysts containing clear liquid (6). Histologically, the cysts of SMAs are composed of simple cuboidal epithelium or flat epithelial cells with clear cytoplasm, but without atypical cells (Table I). Glycogen in the cytoplasm can be demonstrated with PAS staining. These characteristics are important for the differential diagnosis from pancreatic pseudocysts that are composed of inflammatory cells without epithelial cells (7). In addition, the cysts in SMAs are lined by bland epithelial cells that are arranged in a single layer, and MCNs are lined by mucinoustype epithelium (6). The immunohistochemical analysis showed that SMAs are positive for cytokeratins (CKs), neuron-specific enolase, $\alpha$-inhibin and mucin 6 , but negative for neuroendocrine markers such as synaptophysin (SYN), chromogranin A and tyrosine hydroxylase (8). This study showed that the neoplastic cells were positive for CK7 and vimentin (Vim), but negative for CK20 and SYN. However, we are in agreement with the view that an immunohistochemical test is not necessary since the histological appearance of the cells is characteristic (13).

The management of SMAs depends on the symptoms, tumor size and surgical risk (Table I). Resections are reasonable for patients with symptoms or tumors larger than $3 \mathrm{~cm}$ in maximal diameter $(10,11)$. Surgical excision of the mass should be performed when tumor growth is rapid (14). Distal pancreatectomy is nominated when tumors are located in the body or tail of the pancreas. In addition, a Whipple resection is required when the tumor is situated in the head or uncinate process of the pancreas $(13,15)$. It is widely accepted that close follow-up is recommended in asymptomatic patients with tumors less than $3 \mathrm{~cm}$ in maximal diameter, in elderly patients or in those who have high surgical risks. Authors have suggested that follow-up should be scheduled every 6 months for 2 years and annually following that. The follow-up should be continued for at least 4 years, otherwise patients should no longer be considered as candidates for surgical intervention $(13,15)$. Since SMAs have little or no potential of malignant transformation, the prognosis is considered to be favorable. However, long-term follow-up is required for better elucidation of the prognosis of SMAs.

\section{Acknowledgements}

This study was supported by the National Natural Science Foundation of China (No. 30200284, No. 30600278 and No. 30772359), the Program for New Century Excellent Talents in University (NCET-06-0641), and the Scientific Research Foundation for the Returned Overseas Chinese Scholars (2008-889).

\section{References}

1. Compagno J and Oertel JE: Mucinous cystic neoplasms of the pancreas with overt and latent malignancy (cystadenocarcinoma and cystadenoma). A clinicopathologic study of 41 cases. Am J Clin Pathol 69: 573-580, 1978.

2. Morohoshi T, Held G and Kloppel G: Exocrine pancreatic tumours and their histological classification. A study based on 167 autopsy and 97 surgical cases. Histopathology 7: 645-661, 1983.

3. Subramanian S, Marappagounder S, Selvaraj DR and Elangovan B: A rare association of serous cystadenoma of the pancreas with mediastinal lipoma: a case report. Cases J 2: 7165 , 2009.

4. Sand J and Nordback I: The differentiation between pancreatic neoplastic cysts and pancreatic pseudocyst. Scand J Surg 94: 161-164, 2005.

5. Tanaka M, Chari S, Adsay V, et al: International consensus guidelines for management of intraductal papillary mucinous neoplasms and mucinous cystic neoplasms of the pancreas. Pancreatology 6: 17-32, 2006.

6. Goldsmith JD: Cystic neoplasms of the pancreas. Am J Clin Pathol 119 Suppl: 3-16, 2003.

7. Ng DZ, Goh BK, Tham EH, Young SM and Ooi LL: Cystic neoplasms of the pancreas: current diagnostic modalities and management. Ann Acad Med Singapore 38: 251-259, 2009.

8. Kosmahl M, Wagner J, Peters K, Sipos B and Kloppel G: Serous cystic neoplasms of the pancreas: an immunohistochemical analysis revealing alpha-inhibin, neuron-specific enolase, and muc6 as new markers. Am J Surg Pathol 28: 339-346, 2004.

9. Warshaw AL, Compton CC, Lewandrowski K, Cardenosa G and Mueller PR: Cystic tumors of the pancreas. New clinical, radiologic, and pathologic observations in 67 patients. Ann Surg 212: 432-443; discussion 444-445, 1990.

10. King JC, Ng TT, White SC, Cortina G, Reber HA and Hines OJ: Pancreatic serous cystadenocarcinoma: a case report and review of the literature. J Gastrointest Surg 13: 1864-1868, 2009.

11. Tseng JF, Warshaw AL, Sahani DV, Lauwers GY, Rattner DW and Fernandez-del Castillo C: Serous cystadenoma of the pancreas: tumor growth rates and recommendations for treatment. Ann Surg 242: 413-419; discussion 419-421, 2005.

12. Pyke CM, van Heerden JA, Colby TV, Sarr MG and Weaver AL: The spectrum of serous cystadenoma of the pancreas. Clinical, pathologic and surgical aspects. Ann Surg 215: 132-139, 1992.

13. Garcea G, Ong SL, Rajesh A, et al: Cystic lesions of the pancreas. A diagnostic and management dilemma. Pancreatology 8: 236-251, 2008.

14. Vernadakis S, Kaiser GM, Christodoulou E, et al: Enormous serous microcystic adenoma of the pancreas. JOP 10: 332-334, 2009.

15. Sahani DV, Saokar A, Hahn PF, Brugge WR and Fernandez-Del Castillo C: Pancreatic cysts $3 \mathrm{~cm}$ or smaller: how aggressive should treatment be? Radiology 238: 912-919, 2006. 